\title{
Wireless Technology in Medicine Applications
}

\author{
Otto Dostal and Karel Slavicek \\ Institute of Computer Science \\ Botanicka 68a, 60200 Brno, Czech Republic, \\ \{otto, karel\}@ics.muni.cz \\ http://www . ics.muni.cz
}

\begin{abstract}
Institute of Computer Science of Masaryk University is working on the field of supporting medicine multimedia data transport archiving and processing more than ten years. Since first steps like transport of ultrasound and CT images across private fibre optics network these activities have grown up to regional PACS archive. Today more and more hospitals are participating on this project know under the name MediMed. For deployment of MediMed to some remote locations and especially to residence users (medicine specialists) it was necessary to utilise wireless and microwave technologies. Experiences with usage of wireless technology for delivery of medicine picture data are described in this paper.
\end{abstract}

Keywords: PACS, DICOM, Medicine Picture Data

\section{Introduction}

Institute of Computer Science of Masaryk University operates shared regional PACS (Picture Archiving and Communication System) since 2002. That solution was from its start designed to serve as a reliable and accessible communication node and also as an educational and research centre available to any hospital or other healthcare institution, including medical faculties, interested in participating.

The aim of our project was to establish a collaborative platform supporting daily routine in radiology community, to develop a communication channel supporting the exchange of information and special consultations among various medical institutions and also to support medical training for practicing radiologists and medical students. We enable the users from outside the hospitals to have the same access and functionality allowing them to have almost the same working conditions as in the radiology departments of their hospital.

One of the goals of our solution is also to establish an open, collaborative environment to support coordinated research and education among cooperating health care institutions and faculties of medicine by exploiting the large potential of databases of medical image information being processed in hospitals today.

Please use the following format when citing this chapter:

Dostal, O., Slavicek, K., 2007, in IFIP International Federation for Information Processing, Volume 245, Personal Wireless Communications, eds. Simak, B., Bestak, R., Kozowska, E., (Boston: Springer), pp. 316-324. 


\section{PACS}

The PACS (Picture Archiving and Communications System) is a currently used procedure and methodology for processing medical multimedia data obtained from picture acquisition machines like computer tomography, ultrasound, x-ray etc. Multimedia medicine data obtained from these machines (in PACS terminology called modalities) are stored in central PACS server. The PACS server then provides these multimedia data to viewing stations. Viewing stations serve to radiologists for analysing the multimedia data. This approach offers much more capabilities than former film medium. Viewing stations allow image transformation, combination of images from more modalities etc. National Electrical Manufacturers Association (NEMA) has developed a standard for communications between modalities, PACS servers and viewing stations. This standards is DICOM. Currently DICOM version 3.0 is used in mostly all modalities and PACS servers. The structure of PACS is cleanly presented on the figure 1.

Fig. 1. Common structure of PACS system. Modalities serve for acquisition of medicine multimedia data. These data are stored in PACS server and examined and analysed in viewing stations.

The system deals with transmitting, archiving, and sharing medical image data originating from various medical modalities (computer tomography, magnetic resonance, ultrasound, mammography, etc.) from hospitals. The central PACS serves as a metropolitan communications node as well as a long term archive of patients' image studies [7]. The technological solution is described in [4] and [6]. Deep theoretical background of PACS principle and int basic protocol DICOM is discussed in [1] and [2].

\section{MediMed}

Today most hospitals are using local PACS system serving only to one hospital. The goal of the MediMed project [5] is to start a collaboration among hospitals with respect to archiving and use of medical multimedia data and to provide the necessary technological infrastructure for the System.

The realisation of the project facilitates fast communication among individual hospitals, allows decision consultations, and brings various other advantages due to direct connections via optic networks. In general the MediMed project is clearly designed to support society-wide healthcare programmes in the Czech Republic as well as programmes implemented by other countries. The system is also supposed to serve as a learning tool for medical students of the Masaryk University as well as physicians in hospitals.

The gradual development of the joint system for processing and archiving image information is a natural step towards an increasing health care standard 
in the city of Brno and the whole region. Information on a patients treatment in his own healthcare centre as well as in other centres would be available. Consultations by more specialists will be enabled over the patent's picture, in case that a required specialist is not available in the centre in question. Image information evaluation can be carried out in another place, general practitioners in the country will be able to consult specialists in hospitals, etc. Examination results will be available for the doctors in much shorter time than before.

Outsourcing of the hospitals' archiving and communications technology permits cooperation among hospitals and usage of existing patient multimedia data. The Shared Regional PACS is more than just a set of computer network applications. Gradually, it changes the thinking of medical specialists and gets them to cooperate and share data about patients in electronic form. It builds a network of medical specialists. The impact of this work is not only in patient care but also in the education of medical specialists. The implementation of the project has increased the speed of communication among individual hospitals, allowed decision consultations, and brought various other advantages due to dedicated network connections.

\section{Technology background}

The main idea of private network serving for regional PACS archive is the following: We put our firewall in front of a hospital's router or firewall. Our firewall is connected to PACS server's firewall via any secure way. In the case of hospital reachable via private fiber optics network of Masaryk university a dedicated fiber optics pair is used. For remote hospitals the most common way is to use IPSEC tunnel on top of any public data network - in most cases the Internet.

This approach allows us, as administrators of the application, to control the access to central resources and allows the administrators of the hospital's network to control the access to their network. That way everybody can control access to the part of the network that he is responsible for. The situation is easy to see from figure 2 .

Fig. 2. PACS connectivity for hospitals reachable via university private fiber optics network

There is a large fibre optic cable network owned and operated by the universities in the city of Brno. The development of this network started in 1993. It is a private network of all Brno universities connected to the National Research and Education Network (NREN), operated by CESNET association, through the Masaryk University computer centre. The network connects all universities in the city and their faculties spread around the city, various departments of the Academy of Science, most hospitals and some other institutions. Currently the network consists of about $100 \mathrm{~km}$ of optic cables and more than 100 nodes are 
using it. Since the Brno Universities own the fibre optic network, they can ensure that there is sufficient fibre optic cable available to implement new applications and to support new initiatives. The ownership provides the freedom to establish private connections dedicated to these applications.

For hospitals beyond the scope of university's optocable network we need to use public data network. The usage of the public data network forces us to use strong cryptography to secure the tranported data. For this purpose we use IPSEC with AES or in case of equipment with pure CPU performance 3DES encryption algorithm. The logical view of intercity connections to central PACS archive is in figure 3 .

Fig. 3. Remote hospitals interconnection.

Since Regional PACS system is used on a regular production basis it should provide users with reliable and safe services. Because we are dealing with very sensitive information we strongly relay on data storage and transport security. Regional PACS archive is running on dedicated network infrastructure with mostly no interaction with another data networks. In case that it is necessary to use public data networks for servicing remote hospitals we are using strong cryptography for securing medicine data transport.

As the MediMed project was growing up it was necessary to duplicate critical components into remote location to provide required reliability and availability of medical data. The regional PACS system is situated in two locations. The distance between them is about $3 \mathrm{~km}$. All crutical data are stored in both locations. there are used PACS servers from different vendors to have backup for possible (however not probable) breakdown of server system vendor and especially services supported by that vendor. The overall situation is illustrated on picture 4 .

Fig. 4. For reliability improvement there exist backup servers in other location. Backup servers are about $3 \mathrm{~km}$ away from primary ones.

Medicine multimedia data processing is based on DICOM standard. In the case of a single hospital, the problems of user authentication and authorisation are easily solved. In the case of the network with several hospitals, authorisation can be for instance based on the IP address of the requesting computer station. This approach is acceptable in the case of medical modalities and computer stations used by a single person. With greater use of the metropolitan grade of PACS there is the problem of several users sharing the same PACS viewing station. This problem is solved by the use of IPSEC. All users sharing the one PACS viewing station use IPSEC for communication with the central PACS 
archive. After successful authentication, the IPSEC server gives each user a unique private IP address to the endpoint of the IPSEC tunnel. This allows us to use IP based authorisation for access to medical data. Because authenticated users of the PACS archive need to change locations several times a day, it is necessary to provide a fast and easy method of authentication while maintaining a high level of security. For these reasons we use the Public Key Infrastructure (PKI) authentication with the private key stored on a USB key. The keys are stored in the certification authority (CA) operated by Masaryk University.

The amount of various equipments used in this project is increasing during its development and deployment of new functionality. Recently the number of used devices enforced development of centralised management system. This system provides all the necessary supplemental services like collecting of traffic statistics, backup of configurations, time synchronisation, authentication of network managers, etc.

Two Linux based servers are used as central management stations. These stations form redundant solution for all the goals listed above. The primary management station is located at the Institute of Computer Science of Masaryk University and the second one is located at the Faculty of Medicine. Each management station provides full set of services and both stations work independently.

The open source solution - Nagios - was selected for network status monitoring. This software is used for monitoring of network component status, CPU utilisation number of active users, number of running processes, local disk usage of servers and also monitoring of disk usage of central storage system. The whole monitoring system is accessible via web interface and provides the current status as well as the history of all equipments and services availability. Critical alarms are propagated via SMS messages distributed via SMS terminals Siemens 35i connected via RS232 interface directly to both management servers.

\section{Wireless technology}

As already mentioned, the backbone system uses optical wires as a transport medium. Nevertheless, only the hospitals in the city and several of the others in the republic are connected by optical wires.

Another transport medium which is being used is the radio connection. It may be utilized for the main connection of the locality, however in that case the bigger hospitals require at least $20-30 \mathrm{Mbit} / \mathrm{s}$ speed. We are speaking mainly about sending and storing pictures from MR, CT, and such where there is a high demand for transport capacity.

Radio connection is also being used as the so called last mile. We have optical connections between cities, but the radio connection is needed to connect the hospitals inside the city. This concerns establishing traffic in the paid band. Another large area where we employ radio connection is to provide a backup connection. In that case, the requirements for data capacity are lower than for 
the main connection, however, they still must be sufficient for the operation of the medical facilities.

One of the biggest groups of the users of the wireless networks are the radiologists. They very frequently use the opportunity to create the descriptions of the pictures at home. That way, they may react to urgent cases immediately. There is no need to go to hospital and start working on a pressing case after a significant delay. Simultaneously, they save their time, because the work on the picture may often take significantly less of it, than the voyage. The map of wifi coverage of city is on figure 5 .

There are some issues with the usage of the wireless systems. The performance may drop during bad weather such as heavy snowing and there are sometimes problems in the cities when a new building (or an extension of an old one) emerges in the way of the original signal. Still, it can be said, that their employment accelerates the exploitation of systems for archiving, exchange and processing of data as a whole.

Fig. 5. WIFI coverage of the city of Brno. The black triangles are home offices connected via university's private wifi network.

The way of usage of these ways to transfer data is different in different medical facilities. One of the possibilities is, that the doctors have radio connections to their houses, so they may describe pictures at home, consult over medical information with a specialist in another medical facility in another city (or even state), etc. Also, the cable television connections are being used for this purpose if the doctors have them.

As for the doctors working outside their workplace, there is a frequent request. for a transportable notebook with mobile telephone for a connection to the central system. However, the limited speed available limits the range of modalities to work with.

Another technology, which we use for the MediMed project, is the satellite. Within the scope of HEALTHWARE project (which is a 6th EU framework program project), there are being installed terminal satellite devices to the places, where any proper connections are not existent. Some facilities, such as medical institutions for patients with tuberculosis, may be found in woodlands, areas without industrial burden. Than, the usage of a satellite system is one of the few ways, we may use for transfer of a medical information. It is therefore used despite its limited data capacity, that is so needed in the cases of urgent demands for transfer and processing of medical image data.

\section{International collaboration}

We cooperate it the area of processing of medical image information also in international scale. For example the Healthware (Standard and interoperable 
satellite solution to deploy health care services over wide areas) project within the sixth framework programme of EU covers many telemedicine activities.

The goal of this project is developing of healthcare services over the satellite network to increase quality and comfort in European medical practices. The aim is to bridge the medical digital divide in Europe by designing, integrating and validating interoperable telecoms and services platforms to provide existing and future health care services. The satellite based platforms can interact with mobile and terrestrial technologies to supply effective and reliable end-to-end healthcare services and boost the deployment of large-scale satellite communications telemedicine services.

Additionally, Healthware will have a beneficial effect on training and education as far as 7 Universities and Research Centres are concerned. For undergraduate, post-graduate and $\mathrm{PhD}$ students, the participation in such programs is a unique opportunity to be exposed to team work with regular reporting and evaluation by the partners. The research performed is usually of very high quality due to the number experts involved in the group and the concentration of financial resources. It is also the occasion to be exposed to a multicultural environment and to establish international relationships that are very useful to build and strengthen the European Research Area.

\section{Activities supporting medical training}

Teaching has always been one of the most important parts of radiology. Nurture an excellent radiologists in this technological age involves more resources, new methodologies, reorganization of radiological training.

The core of our solution is tailored PACS. That PACS can be used as a "PACS trainer" for students and young radiologists but also forms the basis for additional educational and research applications such as for example the Case Studies describing treatment of real patients. The Case Study is an integrated hypertext document forming didactic unit and consists of short texts, structured clinical data, radiological images of various kinds, images from nuclear medicine modalities, macroscopic and microscopic pathology images or demonstration of the video movies recorded during surgeries.

Images appropriate for teaching and research purposes are made anonymous (i.e. the personal data of the patient and other information that may disclose the identity of the patient is replaced with fictitious information or modified in such a way so as not to lose any relevant information but so as to prevent disclosure of the patients identity) when sending into Educational and Research PACS.

One of the basic principles when sending images into the Educational and Research PACS is the coordinated assignment of fictitious patient identity, so it can offer a more complex view of the evolution of the patients health in situations where the patient is being treated in different healthcare facilities. Therefore, the legal barrier preventing access to sensitive and confidential patient data is removed. 
The Case Study can be accessible via standard web browser and if the users have DICOM diagnostic workstation installed on their computers, then the referenced image study can be manipulated and processed in all ways supported by the particular workstation. It means that medical students can access large amounts of systematized medical cases related to their subject. The labs equipped with appropriate software can also serve as training simulators for those training to be radiologists. The students can learn more practical lessons instead of wasting their time in the library.

\section{Conclusion}

The efficiency and rationalization of technological and human resources must be considered in connection with improvements of the quality of healthcare. Teleradiology in the Czech Republic is crucial because medical experts and specialists can be available in urgent cases permitting qualified external medical experts to be involved in the diagnosis. Regional-level procurements of systems and services are preferred to achieve major savings and improve the quality of healthcare including the learning processes.

The evolution of educational and research services provided by this solution is also influenced by emerging wireless communication technology. This technology permits the appropriate services to be accessible also through the satellite network covering the Europe. The satellite based platforms can interact with mobile and terrestrial technologies.

\section{References}

1. K.J.Dreyer- D.S.Hirschorn-J.H.Thrall- A. Mehta , PACS A Guide to the Digital Revolution, 2006, Springer Science +Business Media, Inc., USA, ISBN 978-038726010-5

2. H. K. Huang, DSc, PACS and Imaging Informatics: Basic Principles and Applications, Hoboken, NJ: Wiley, 2004 ISBN 0-471-25123-2.

3. M. Petrenko - P. Ventruba - O. Dostal, First application of Picture Archiving and Communication System (PACS) in Gynecologic Endoscopy, ISGE - 6th Regional Meeting, Bangkog. ISGE 2002, p.53-56.

4. O. Dostal - M. Filka - M. Petrenko, University computer network and its application for multimedia transmissin in medicine, WSEAS Int. Conf. on Information Security, Harware/Software Codesign, ECommerce and Computer Networks, Rio de Janeiro, Brazil; WSEAS 2002, 1961-1964

5. O. Dostal - M. Javornik - K. Slavicek, MEDIMED-Regional Centre for Archiving and Interhospital Exchange of Medicine Multimedia Data, Proceedings of the Second IASTED International Conferee on Communications, Internet and Information Technology. Scottsdale, Arizona, USA : International Association of Science and Technology for Development- IASTED, 2003. ISBN 0-88986-398-9, p.609-614.

6. O. Dostal - M. Javornik - K. Slavicek - M. Petrenko - P.Andres, Development of Regional Centre for Medical Multimedia Data Processing, Proceedings of the Third IASTED International Conferee on Communications, Internet and Information Technology. St.Thomas, US Virgin Islands: International Association of Science and Technology for Development- IASTED, 2004. ISBN 0-88986-445-4, p.632-636. 
7. M. Schmidt - O. Dostal - M. Javornik, MEDIMED - Regional PACS Centre in Brno, Czech Republic, Proceedings of the 22th International Conference of EuroPACS \& MIR (Managenment in Radiology) Conference, 16 - 18 September, Trieste, Italy

8. Dostal O. - Javornik M.: Regional Educational and Research Centre for Processing of Medical Image Information, CARS 2005 Computer Assisted Radiology and Surgery, June 22.-25. 2005, Berlin, Germany, p.911-915. ISBN 0-444-51872-X, ISSN 05315131. 